\title{
Varia.
}

\section{Ernest Besnier.}

Die Dermatologie verliert in der Person von Ernest Besnier einen ihrer allgemein bewunderten und geachteten Vertreter.

Geboren im April 1831, wurde er, in seiner glänzenden medizinischen Karriere, in rascher Aufeinanderfolge 1853 zum Assistenzarzt der Pariser Spitäler, 1873 zum Arzt daselbst und 1881 zum Mitglied der Akademie der Medizin ernannt. Seit seinem Eintritt in das Hôpital St. Louis als Abteilungsvorstand, im Alter von 42 Jahren, widmete er sich ganz dem Studium der Hautkrankheiten. Nach einem Stadium des Herum tastens, das jeder Arzt, selbst der Kenntnisreichste, der sich mit dieser speziellen Pathologie befaßt, durchmachen muß, wußte er bald seine Persönlichkeit zu behaupten und vor allem sich dem damals maßgebenden Einfluß Bazins zu entziehen. In späteren Jahren selbst Haupt einer Schule, gab er seiner ganzen Spitalskarriere den Stempel angestrengtester, wissenschaftlicher Tätigkeit und setzte als Kliniker, Verbreiter und Organisator stets seine ganze Persönlichkeit ein. Seine klinischen Vorlesungen waren die meist besuchtesten, sowohl von französischen Medizinern und Ärzten, als von ausländischen Dermatologen, die beständig dem schönen und lehrreichen Hôpital St. Louis zuströmten, und von seinen ehemaligen Schülern, die stolz darauf waren, unter ihm gedient zu haben. Seine Werke waren sehr zahlreich und bedeutend. Vor allem ist die Übersetzung der Vorlesungen Ka p o s is zu nennen und die damit zusammenhängenden erklärenden Anmerkungen. Man findet darin kampfbereite Aufstellungen seiner eigenen Schule, die diejenigen des bedeutenden Wiener Meisters ergänzen oder ihnen widersprechen. Zwei Auflagen dieser Vorträge waren mehreren medizinischen Generationen in Frankreich und anderen Ländern beim Studium der Hautkrankheiten von großem Nutzen.

Ernest Besnier blieb dabei nicht stehen. 1900-1904 veröffentlichte er mit B rocq und Jacquet das große Werk "La pratique dermatologique", ferner nahm er regsten Anteil an den Arbeiten der 1888 gegründeten Vereinigung der Ärzte vom Hôpital St. Louis und von 1889 an, an denen der französischen Gesellschaft für Dermatologie und Syphilis, deren Präsident er war. Von 1893-1902 immer wiedergewählt, leitete 
er die Debatten mit einer Entschlossenheit und Scharfsichtigkeit, die die Bewunderung seiner Zuhörer erregte.

Ernest Bes nier gab auch den Ansto 3 zu dem ersten internationalen Kongreß für Dermatologie und Syphilis, der 1889 in Paris stattfand und dessen Organisator er war. Ebenso nahm er einen bedeutenden tätigen Anteil an dem Londoner Kongreß 1896 und leitete im Jahre 1900, der Blütezeit seiner medizinischen Karriere, als Präsident den II. Pariser Kongreß. Er hat endlich als Chef der französischen Delegation zum Erfolge der Lepra-Konferenz, welche 1897 in Berlin stattfand, viel beigetragen. Allen denjenigen, die an diesen bedeutenden Versammlungen teilgenommen haben, wird die Erinnerung an die starke Macht seiner Worte, die sich im richtigen Moment bis zur Beredsamkeit steigerte, ebenso nnvergeßlich bleiben wie seine Anziehungskraft, das leutselige Entgegenkommen, dessen alle Lernbegierigen bei ihm sicher waren und sein würdevolles Wesen.

Eine gutgelungene Denkmünze verewigt seine Züge, gibt aber leider nur eine schwache Vorstellung seines feinen, scharfen, durchdringenden Blickes und der offenen, oft lächelnden Gesichtszūge, die die seltenen geistigen Eigenschaften verrieten.

Der Name Ernest Besnier wird in der Geschichte der Dermatologie stets lebendig bleiben durch die Erinnerung an sein großes Lehrtalent, seine guten Bücher und seine so zahlreichen interessanten Mitteilungen in der französischen Gesellschaft für Dermatologie und Syphilis, ebenso wie durch Verbreitung der Kenntnis neuer krankhafter Arten und Spielarten.

Ehre seinem Andenken!

H. Hallopeau (Paris).

Personalien. Priv.-Dozent Dr. Brandweiner wurde zum Vorstand einer Hautabteilung an der Wiener Allgem. Poliklinik ernannt. 